\title{
Digital Display Design of Ethnic Clothing of Nanling
}

\author{
Shiyong Zheng ${ }^{1, a}$, Weili Guan ${ }^{\star 2, ~ b}$, Biqing $\mathrm{Li}^{3, \mathrm{a}}$ \\ ${ }^{1}$ College of Computer Science and Information Engineering, Hezhou University, Hezhou Guangxi \\ 542899, China \\ ${ }^{2}$ College of Information Engineering, Nanning University, Nanning Guangxi 530200, China \\ ${ }^{3}$ College of Mechanical and Electronic Engineering, Hezhou University, Hezhou Guangxi 542899, \\ China \\ ajanliful@163.com, ${ }^{\mathrm{b}} 13508326 @ q q . c o m$
}

Keywords: Ethnic clothing of Nanling; Mobile terminal; Three-dimensional modeling; Digital platform

\begin{abstract}
This paper conducts a research about digital display of “ Nanling minority costumes” of Hezhou University Museum. Sufficient basis for the work in the feasibility study and needs analysis, a digital display design is proposed. This design refers to clothing patterns design process, user interface design and three-dimensional modeling process in digital display platform. Through the platform, ethnic culture can be better displayed, making visitors gain better user experience.
\end{abstract}

\section{Needs Analysis of Nanling Ethnic Clothing Digital Display Platform}

The Advantages of Nanling Ethnic Clothing Displayed on Mobile Terminal.

Nanling region contains several ethnic minorities, including Yao, Zhuang, Miao, etc. Different ethnic groups cause differences on clothing. If people can directly visit relics by using smart-phones, besides, they only need to lightly click on phone screen to zoom in or out the displayed relics, then they can have a better understanding of details of relics, it will help to strengthen protection of relics and increase people's interest and understanding of relics.

\section{Needs of Technology Equipment Development of Digital Museum.}

First of all, developing a digital museum not only needs rich heritage exhibits and related relic materials as basic sources but also can update their changes, and gradually enlarges exhibits, by which the effectiveness of cultural transmission is enhanced.

Furthermore, now that the exhibits are open, there must be full-featured network data collection platform. The database contains images, sounds and colors and by this system, information of relics and their features are transfered to network, thus, visitors and anyone interested in them are offered Wi-Fi service to get a better understanding of related museum information.

Finally, Internet information and that on mobile terminal can mutually exchange, for example, while appreciating a works, people can use QR code of smart-phone to scan it to get its information. With the help of Beacon technology, words introduction set up at pavilion directly reach mobile client.

\section{Design of Digital Display of Nanling Ethnic Clothing}

\section{Interface Design of Digital Display of Nanling Ethnic Clothing.}

Flat style is mainly applied in digital display design. However, different minorities have different styles and each has its own advantages. In this case, flat style and three-dimensional modeling are combined in the design.

1) Programming Language Design

With the diversity of mobile terminal software and development of intelligent phone, exhibits are much more various in kind but more difficult in manufacturing. Therefore, analyzing, filtering and integrating relic data in time is needed so that diverse Icon with different sizes are designed, which improves efficiency. (eg.picture1) 


\section{2) Interface Design}

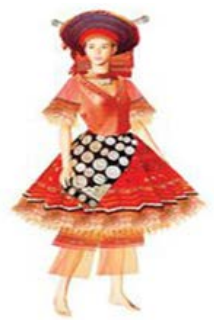

eg.picture1

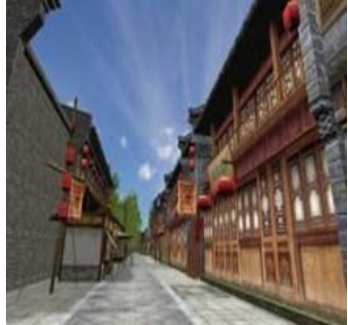

eg.picture2

The realism of three-dimension is applied in interface design to provide visitors with a kind of immersive feeling. Photoshop software is made use of in the design, whose most important characteristic is bringing good overall effect such as harmony degree of color, layout of scene, the intensity of the light and the the selection of role and so on. Different distances, volumes and places make it hard for visitors to feel the relics thoroughly so the designer should not only correctly express the contents of relics with brief and easy words, but also should put himself in visitors' shoes.(eg.picture2)

3) Scene Design

Each nation changes its living style in accordance with topography, climate and habits. Take Fengyuqiao of Dong as an example. It combines landscapes, natural beauty and local weather in design. And Diaojiaolou whose functions are moisture-proof ,ventilation,and anti-animal. It can also protect limited local resources and the fragile ecological environment. While designing costumes, unique symbols of ethnic building can be added as background to increase the vividness.

4) Role Design

Just as the proverb goes "“The unique features of a local environment always give special characteristics to its inhabitants." With the prosperity and development of culture, nations communicate with each other. According to color,there are four kinds of races namely the yellow, the black, the brown and the white. And the descendants of the Chinese nation are called "yellow". Each nation has its own representatives. Different people from different nations have different appearances but they have something in common as well. For example, Zhuang people have approximate bronzed skin and strong body.(eg.picture3)

5) Costume Design

Costume design can embody a person's spirit, while ethnic clothing shows not only the spirit, but also characteristic of a nation. To take Yao as an example, both men's and women's clothing have their own characteristics. Generally speaking, Women wear dark collarless shirt, and lighter collar of the shirt wore inside turned on neckline, matching cloth shoes and trousers. However, Yao people live scattered, women's clothing are different. Some take white silk rope as match inside and outside colored ribbons wrapped, and embroidered cloth on the top. Men's clothing is much easier than women's. The differences of Yao clothing show the significant meaning of clothing design. Different nations have different headwears. Each nation has its own symbolic symbol. Eg, Miao takes horn comb totem as its symbol; Huang Ping Miao's headdress are mainly phoenix image, symbolizing that Phoenix is the queen of all birds. The differences of Headwears also mean different ages in some nations. older women wear blue triangle hat, meaning a long life while young girls wear triangle hat made of flower cloth symbolizing a great future. All in all, designing clothing should take characteristics of Nanling nations into consideration. Spare every effort to show clothing culture of Nanling nations to attract more and more visitors. (eg.picture4)

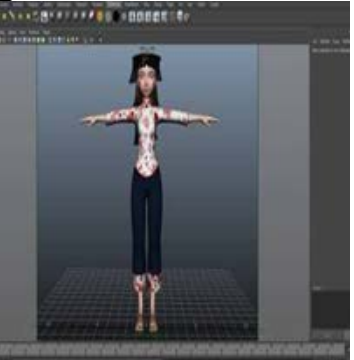

eg.picture3

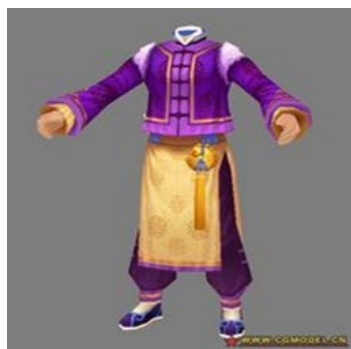

eg.picture4 


\section{Three-dimensional Modeling.}

This top mainly takes Nanling ethnic clothing as an example to make Nanling national culture be understood. To this point, habitability, interestingness, participation and interactivity should be paid more attention to in the process of display. Eg a full range of simulation of Yao national clothing needs to know its style, the overall effect and material selection, which thoroughly embodies characteristics of Yao clothing and let people know more about this ethnic clothing and national culture.

\section{Implementation of Digital Display of Nanling Ethnic Clothing.}

1) Virtual Reality Design

Virtual reality technology,involving a wide range, has a realistic three-dimensional sense of vision, hearing, and smell, making people, the participants involuntary experience the virtual world by appropriate means. Reforming and updating the simulated contents in time, visitors can check the contents at any time and anywhere, which not only improves efficiency but also reduces cost.

2) Interactive Technology Design

Interactive technology is a kind of technology that collects a variety of information within a limited area. It is software for tangible carrier text message. Factors such as distance and setting place prevent visitors from getting the feeling as that when they are visiting. However, interactive design can show every detail of clothing. It is totally possible for visitors to appreciate the clothing at a very close distance by making use of their imagination and operating the computer software. Furthermore, configuring music or installing or reinstalling games for the chosen roles is possible as well.

\section{Conclusion}

This paper takes "Nanling ethnic clothing" as the top, describing 3D digital design, combining developing demand of Nanling ethnic clothing, completely analyzing and stating Nanling ethnic clothing from different angles and technical levels, which will make national clothing have a sound inheritance and development, and make more people gain different experience of historical relics.

This work is supported the following fund:

Nanling corridor of ethnic culture research base of Open Fund Project: "Nanling National corridor costumes digital display design" (No 2015kf27);

Project of scientific research and technology development project of Hezhou: "Design and implementation of agricultural products e-commerce platform based on Semantic Technology” (No, Hekeneng 1506006)

Guangxi science and technology development plan (No 11107006-13 \&\&No 12118017-10A); Nanning scientific research and technology development project(No 20121033);

Nanning science and technology development plan project of Yongning district (No 20130332D);

Scientific research project of hezhou university: "The intelligent home system Implementation Research based on the Internet of Things technology" (No 2014YBZK10); "Research and development of E-government platform based on mobile terminal” (No 2015ZZZK03);

Reform in Education project of hezhou university: "Internet of things"(No hzxytszy201501); "Research on application personnel training model of Communications for SMEs"(No hzxyjg201525);

Master degree discipline construction scientific research and Cultivation Project: "Research on the social work service of the teenagers' Network Addiction"(No 2015SHGZ005) \&\&"Research on the intelligent development of rural tourism in Guangxi based on the Internet of things" (No 2015MTA16); 


\section{References}

[1].Wan Yonglin, on the protection and development of the intangible cultural heritage of Yunnan ethnic minorities,Journal of Kunming University of Science and Technology [J] 2012 (6)

[2].Gao Xing, Research on the design of Minority Costume Display in the context of cultural diversity, Journal of Chongqing University of Science and Technology, [J]2012 (12): 159-163

[3].Feng Weixia, the application of new media in the digital display of the museum, the concept of [J]2015 (3), 76-78 